\title{
Recanalization of Emergent Large Intracranial Vessel Occlusion through Intravenous Thrombolysis: Frequency, Clinical Outcome, and Reperfusion Pattern
}

\author{
Carmen Serna Candel $^{\mathrm{a}} \quad$ Marta Aguilar Pérez $^{\mathrm{a}}$ Victoria Hellstern ${ }^{\mathrm{a}}$ \\ Muhammad AlMatter ${ }^{a}$ Hansjörg Bäzner ${ }^{b}$ Hans Henkes ${ }^{a, c}$

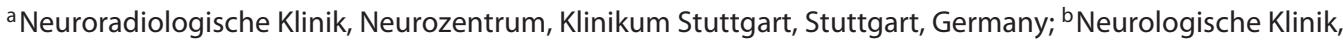 \\ Neurozentrum, Klinikum Stuttgart, Stuttgart, Germany; ' ${ }^{C}$ Medical Faculty, University Duisburg-Essen, Essen, Germany
}

\section{Keywords}

Intravenous thrombolysis · Large vessel occlusion .

Reperfusion · Endovascular treatment

\begin{abstract}
Background: According to a recent meta-analysis, 1 out of 10 patients with emergent large intracranial vessel occlusion (ELVO) causing stroke have recanalization after intravenous thrombolysis (IVT) alone. However, rate, clinical outcome, and recanalization pattern of this phenomenon are poorly understood. Objectives and Methods: Patients with ELVO recanalized only by IVT were analyzed, and frequency of recanalization, clinical outcome, safety variables, and reperfusion pattern were assessed. These patients were compared to a group of patients with ELVO who underwent endovascular thrombectomy with or without prior IVT. $\boldsymbol{R e}$ sults: Successful or sufficient recanalization after IVT alone occurred in 81 of 760 patients (10.6\%) with ELVO who had been referred for endovascular thrombectomy. These 81 patients (group 1) were compared to a group of patients receiving endovascular thrombectomy with prior IVT (group 2 ) or without (group 3). A good clinical outcome at 90 days was seen in $61.7 \%$ of patients in group 1,32.2\% in group 2 , and $34.5 \%$ in group $3(p<0.001)$. The 3 groups had no sig-
\end{abstract}

nificant differences in intracranial hemorrhage. IVT was not independently associated with symptomatic intracranial hemorrhage, parenchymal hematoma, or subarachnoid hemorrhage. Mortality at 90 days was $9.9 \%$ in group 1, $20.7 \%$ in group 2 , and $29.6 \%$ in group $3(p<0.001)$. After adjusting for all relevant variables, outcome and mortality differences were nonsignificant. No difference in the rate of successful reperfusion (modified treatment in cerebral ischemia $[\mathrm{mTICl}] 2 \mathrm{~b} / 3$ ) was found. A reperfusion $\mathrm{mTICl} 3$ was achieved in $18.5 \%$ in group $1,60.7 \%$ in group 2 , and $57.1 \%$ in group $3(p<0.001)$. Patients in group 1 had lower chance of achieving a complete recanalization $(\mathrm{mTICl} 3)$ compared to patients in group 2, OR $0.15(95 \% \mathrm{Cl} 0.08-0.29)$ and in group 3, OR 0.17 (95\% Cl 0.09-0.32; $p<0.001)$. Conclusions: Primary IVT in ELVO caused a recanalization rate of $10.6 \%$, making endovascular treatment either unnecessary or impossible. Early recanalization of ELVO with only IVT is associated with a $61.7 \%$ independence rate at 90 days and similar successful reperfusion rates ( $\mathrm{mTICl} / 2 \mathrm{~b} / 3$ ) compared to ELVO treated with endovascular treatment, with or without previous IVT. However, recanalization only through IVT achieves a lower rate of $\mathrm{mTICl} 3$ reperfusion when compared to endovascular treatment.

(C) 2019 The Author(s) Published by S. Karger AG, Basel

\begin{tabular}{ll}
\hline KARGER & $\begin{array}{l}\text { @ } 2019 \text { The Author(s) } \\
\text { Published by S. Karger AG, Basel }\end{array}$ \\
E-Mail karger@karger.com & Karger \\
This article is licensed under the Creative Commons Attribution- \\
www.karger.com/ced & $\begin{array}{l}\text { NonCommercial-NoDerivatives } 4 \text {.0 International License (CC BY- } \\
\text { NC-ND) (http://www.karger.com/Services/OpenAccessLicense). } \\
\text { Usage and distribution for commercial purposes as well as any dis- } \\
\text { tribution of modified material requires written permission. }\end{array}$
\end{tabular}

Carmen Serna Candel

Neuroradiologische Klinik, Neurozentrum

University/Hospital Klinikum Stuttgart

Kriegsbergstrasse 60, DE-70174 Stuttgart (Germany)

E-Mail csernacandel@gmail.com 


\section{Introduction}

Patients with an acute ischemic stroke due to emergent large intracranial vessel occlusion (ELVO) are frequently treated by intravenous (IV) thrombolysis (IVT) prior to undergoing mechanical (mTE) and/or aspiration thrombectomy (aTE).

In 1 out of 10 cases, IVT will result in a recanalization of the previously occluded proximal artery, meaning that aTE/mTE will not be necessary or even possible [1]. Little is known about the clinical outcomes and reperfusion patterns of these patients. Our aim is to describe the frequency, clinical outcome, and angiographic pattern of recanalization of this phenomenon and to compare these patients with those on whom aTE/mTE was performed.

\section{Materials and Methods}

\section{Patient Selection}

Using a prospective stroke database, where we collect all the patients referred to our interventional neuroradiology department in order to receive endovascular treatment, we were able to identify $n=3,200$ patients who had been referred for treatment because of an acute ischemic stroke due to ELVO, between February 2007 and January 2019. From this pool, we retrospectively selected patients whose occluded proximal artery had recanalized after IVT, making aTE/mTE either unnecessary or impossible (e.g., due to distal thrombus migration). In total, 35 patients treated in 2007 were excluded because of lack of treatment data with IVT. We calculated frequency of recanalization with only IVT by dividing the number of patients with ELVO that recanalized only with IVT (and did not receive endovascular treatment) by the total number of patients with ELVO that had received IVT. Our hospital is a certified comprehensive stroke center, providing endovascular service for 13 regional stroke units. The local Ethics Committee approved the data collection and analysis.

In this paper, we present the medical history, vessel occlusion as well as clinical and angiographic outcomes of those patients with ELVO for whom recanalization happened following IVT alone (recombinant tissue plasminogen activator [rtPA, alteplase] 0.9 $\mathrm{mg} / \mathrm{kg}$ IV given in standard recommendation). The patients' clinical characteristics were assessed using medical history registries and a modified Rankin Scale (mRS) before stroke. The time of stroke was also noted; if this was unknown, it was defined as the last time the patient was seen well. National Institute of Health Stroke Scale (NIHSS) prior to digital subtraction angiography (DSA) was considered as value for analysis and comparison between the groups. As many patients are transferred to our hospital for mTE from other centers, where they could possibly have received IVT, the initial NIHSS assessment from the referring hospital was compared to the NIHSS result before DSA at our center. A difference of 4 points or fewer was considered nonsignificant. Changes of $>4$ points in NIHSS were deemed early clinical improvement or worsening.
We also carried out a retrospective study comparing the above patients (recanalization following IVT alone, group $1, n=81$ ), regarding clinical and angiographic outcome, with a group of ELVO patients who, during 2016-2017, underwent aTE/mTE without stent implantation, with prior IVT (group 2, $n=122$ ) or without prior IVT (group 3, $n=226$ ). As endovascular treatment technique and materials have evolved during the last years, we chose a consecutive group of patients treated uniformly with a standardized technique (thrombectomy with stent retriever pREset ${ }^{\circledR}$ [phenox, Bochum, Germany] with aspiration or only aspiration with Sofia ${ }^{\circledR}$ [Microvention, Aliso Viejo, CA, USA] or $5 \mathrm{MAX}^{\circledR} \mathrm{ACE}^{\circledR}[$ Penumbra, Inc., Alameda, CA, USA] catheters) in order to make the comparison group more homogenous regarding endovascular technique. Exclusion criteria for groups 2 and 3 were: a mRS of 4 or 5 pre stroke, an Alberta Stroke Program Early Computed Tomography Score (ASPECTS) of 0 as well as tandem occlusion requiring stent-angioplasty or intracranial stenting (either for stenosis or thrombus fixation). We assumed that the comparison group of our real clinical practice to the group of ELVO patients that at the time of the angiography had already a recanalization and reperfusion was not going to be balanced regarding clinical characteristics and imaging variables; this imbalance would influence clinical outcome; for that reason a multivariate analysis was planned.

\section{Imaging, Angiographic, and Clinical Variables}

Vessel occlusion was confirmed in primary imaging (computer tomography [CT]-angiography [CTA] or magnetic resonance-angiography [MRA]). Presence of early ischemic changes was assessed using ASPECTS. Revascularization was assessed with DSA; in group 1, CTA or contrast-enhanced MRA was possible. Angiographic revascularization was assessed using the modified treatment in cerebral ischemia (mTICI) score. This comprises the following values: grade 0 , no perfusion; grade 1 , antegrade reperfusion past the initial occlusion, but limited distal branch filling with little or slow distal reperfusion; grade $2 \mathrm{a}$, antegrade reperfusion of less than half of the occluded target artery previously ischemic territory (or target downstream territory [TDT]); grade $2 b$, antegrade reperfusion of more than half, but less than complete antegrade reperfusion of the TDT; grade 3, complete antegrade reperfusion of the TDT, with an absence of visible occlusion in all distal branches [2]. In group 1, successful or sufficient recanalization was defined as mTICI $2 b / 3$ on the angiography before endovascular treatment or another reperfusion pattern with significant clinical improvement, good collateralization, or impossible endovascular treatment (e.g., due to distal thrombus migration).

Furthermore, for each patient, we noted the following values: time from stroke onset to IVT (time to rtPA), time to intervention (stroke onset up to groin puncture), and time to recanalization (time to end of treatment in groups 2 and 3 and to first assessment of recanalization in group 1). Endovascular treatment was performed with stent retrievers and/or intracranial aspiration as mentioned above.

The clinical outcome was assessed as severity of disability at 90 days according to the mRS. A mRS $\leq 2$ was considered a good clinical outcome [3].

Further rates that were recorded were: mortality at 90 days, subarachnoid hemorrhage (SAH), and parenchymal hematoma ( $\mathrm{PH}$; according to ECASS Classification) in imaging 24-36 h after stroke or symptomatic intracranial hemorrhage (SICH) confirmed 
on neuroimaging (CT or magnetic resonance). A SICH was defined as any type of intracerebral hemorrhage on posttreatment imaging with an increase of at least 4 points on NIHSS (ECASS II) [4].

\section{Statistical Analysis}

Continuous variables were reported as mean with SD or median with interquartile ranges. Fisher's exact test was used for testing the null hypothesis that 2 independent groups do not differ with respect to the distribution of a categorical variable. MannWhitney $U$ test was used to compare whether 2 groups differed regarding the distribution of a metric variable. Logistic regression with forward selection was used to determine which characteristics influence the likelihood of an event happening; only patients with complete data in the relevant variables were evaluated in the model. Comparisons were done between groups 1 and 2 and groups 1 and 3; groups 2 and 3 were not compared. The analysis was performed with Stata/IC 14.2 for Windows, and the level of significance was set at a 0.05 level (2-sided).

\section{Results}

From a prospective stroke database with 3,165 patients with acute ischemic stroke due to ELVO (February 2007 - January 2019), 760 patients (24\%) had received IVT prior to diagnostic DSA and intended intervention (Fig. 1). Out of these IVT patients, 664 patients $(87.3 \%)$ underwent catheter angiography and endovascular treatment because of persistence of the occlusion or incomplete recanalization, and 81 patients $(10.6 \%$, frequency of successful/sufficient recanalization of ELVO with only IVT in our center) underwent catheter angiography where the IVT recanalized the proximal artery making aTE/mTE either unnecessary or impossible (e.g., due to distal thrombus migration). In 15 patients, the endovascular treatment was not performed because of a reason different to recanalization due to IVT: 1 patient with an occlusion of left middle cerebral artery (MCA)-M2 in MRA and transient neurologic deficit declined DSA and eventually an endovascular treatment after being transferred; in 4 of them, DSA was not performed: 2 patients needed cardiopulmonary resuscitation, 1 patient had an already demarcated infarct, one had a chronic occlusion with a small peripheral embolism; in 1 patient, the reason was an acute occlusion with good collaterals that could not be reached endovascularly after a long time; in 8 cases, there were chronic occlusions; 1 patient had a spontaneous recanalization in CTA already before IVT and DSA showed no ELVO.

The $10.6 \%$ rate of successful/sufficient recanalization only with IVT found in our study varies if the patient had been transferred from a referring center where IVT was

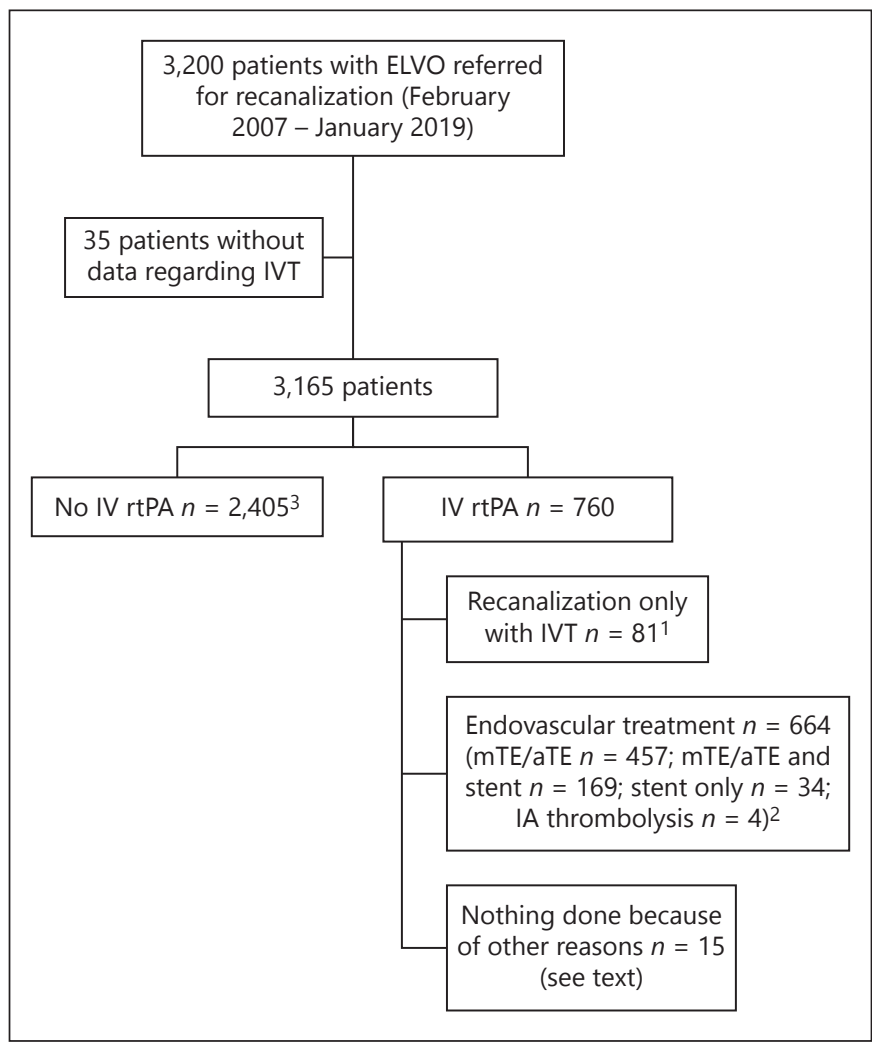

Fig. 1. Flow diagram. ${ }^{1}$ These patients represent group $1(n=81)$. ${ }^{2}$ From these patients, a subgroup of ELVO patients who underwent aTE/mTE without stent implantation, with prior IVT (group 2, $n=$ 122) was selected (see Methods). ${ }^{3}$ From these patients, a subgroup of ELVO patients who underwent aTE/mTE without stent implantation without prior IVT (group 3, $n=226$ ) was selected (see Methods). ELVO, emergent large intracranial vessel occlusion; IVT, intravenous thrombolysis; mTE/aTE, mechanical and/or aspiration thrombectomy; IA, intra-arterial administration; rtPA, recombinant tissue plasminogen activator.

given, $9.3 \%(n=71)$ or if the patient was a direct admission to our center (direct presenter), $1.3 \%(n=10)$ recanalization rate, respectively.

The clinical characteristics of the patients who experienced ELVO recanalization after only having undergone IVT (group 1) are described and compared in Table 1 with the subgroups of patients with ELVO who had undergone mTE/aTE with (group 2) or without previous IVT (group 3 ). The patients in group 1 had lower NIHSS and higher ASPECT scores than groups 2 and 3 and were also younger and with male dominance compared to group 3.

The patients in group 3 had more frequently unknown stroke onset and an mRS $>2$ prestroke and patients in groups 2 and 3 had a significantly longer time to recanalization compared to group 1. 
Table 1. Clinical characteristics

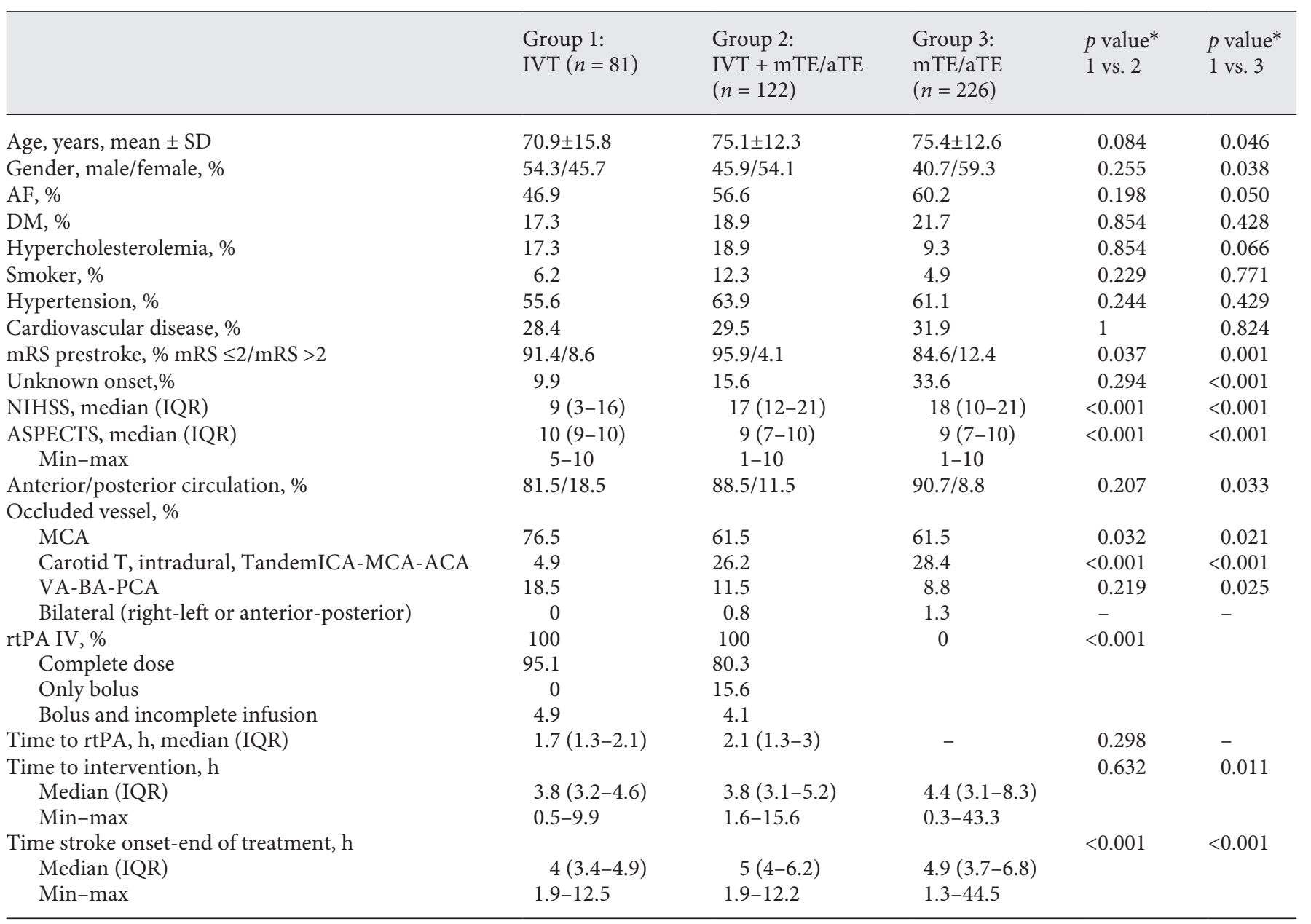

* Fisher's exact test (qualitative variables); Mann-Whitney U test (quantitative variables).

AF, atrial fibrillation; ASPECTS, Alberta Stroke Program Early Computed Tomography Score; DM, diabetes mellitus; IQR, interquartile range; MCA, middle cerebral artery; min-max, minimum and maximum; mRS, modified Rankin Scale; NIHSS, National Institute of Health Stroke Scale; Tandem ICA-MCA-ACA, Tandem occlusion of internal carotid artery and MCA or anterior cerebral artery; VA-BA-PCA, vertebral, basilar, and posterior cerebral artery; IVT, intravenous thrombolysis; aTE, aspiration thrombectomy; mTE, mechanical thrombectomy.

Occluded vessels in group 1 were mostly MCA occlusions, followed by occlusions of posterior circulation (16\% basilar artery and $2.5 \%$ vertebral artery). In groups 2 and 3, most cases were MCA occlusions followed by carotid-T, ICA intradural, or tandem occlusions. MCA occlusions and posterior circulation occlusions were significantly more frequent in group 1 compared to 2 and 3 and carotid-T, ICA intradural, or tandem occlusions in groups 2 and 3 compared to group 1.

Patients in group 1 received complete dose of rtPA in $95.1 \%$. The group 2 dose of IVT was incomplete in $19.7 \%$. In total, $87.7 \%$ of patients with ELVO and recanalization only through IVT (group 1) were transferred to our hos- pital from a referring center where IVT had been given. About $29.6 \%$ had an early clinical neurological improvement, $8.6 \%$ a clinical worsening, $1.2 \%$ had fluctuating improvement rates, and $60.5 \%$ remained clinically unchanged after IVT and after transfer.

Safety variables, clinical and angiographic outcomes are shown in Table 2 . A good clinical outcome $(\mathrm{mRS} \leq 2)$ at 90 days was seen in $61.7 \%$ of patients in group 1, compared to 32.2 and $34.5 \%$ of patients in groups 2 and 3 , respectively $(p<0.001)$. A good clinical outcome (mRS $\leq 2$ ) was significantly associated with male gender, smoking, higher ASPECT score, lower NIHSS, lower time to intervention, and time to recanalization. A worse clinical 
Table 2. Safety parameters, clinical, and angiographic outcome

\begin{tabular}{|c|c|c|c|c|c|}
\hline $\mathrm{SICH}, \%$ & 2.5 & 3.3 & 1.3 & 1 & 0.610 \\
\hline $\mathrm{SAH}, \%$ & 7.4 & 9.8 & 9.5 & 0.792 & 0.809 \\
\hline $\mathrm{mRS}>2$ & 38.3 & 67.8 & 65.5 & $3.39(1.83-6.28)$ & $3.06(1.78-5.27)$ \\
\hline Mortality at 90 days, $n(\%)$ & $8(9.9)$ & $25(20.7)$ & $66(29.6)$ & 0.052 & $<0.001$ \\
\hline mTICI final, $n(\%)$ & & & & 0.252 & 0.656 \\
\hline $0-2 \mathrm{a}$ & $6(7.4)$ & $16(13.1)$ & $22(9.7)$ & & \\
\hline $2 b, 3$ & $75(92.6)$ & $106(86.9)$ & $204(90.3)$ & & \\
\hline mTICI final 3, $n(\%)$ & $15(18.5)$ & $74(60.7)$ & $129(57.1)$ & $<0.001$ & $<0.001$ \\
\hline
\end{tabular}

* Fisher's exact test.

IVT, intravenous thrombolysis; mRS, modified Rankin Scale; mTE/aTE, aspiration and mechanic thrombectomy; mTICI, modified treatment in cerebral ischemia; $\mathrm{PH}$, parenchymal hemorrhage; $\mathrm{SAH}$, subarachnoid hemorrhage; SICH, symptomatic intracranial hemorrhage.

outcome ( $\mathrm{mRS}>2$ ) was significantly associated with age, unknown stroke onset, $\mathrm{PH}$, and $\mathrm{SAH}$. After adjusting for all the relevant variables in the multivariate analysis, the difference in clinical outcome between group 1 and groups 2 and 3 was no longer significant (Table 3a).

With regard to safety variables, $\mathrm{SICH}$ was $2.5 \%, \mathrm{PH}$ $5.8 \%$, and SAH $7.4 \%$ in group 1 . There were no significant differences in these variables in groups 2 and 3 , where the values were $3.3,5.7$, and $9.8 \%$ and $1.3,6.3$, and $9.5 \%$, respectively. IVT in groups 1 and 2 was not independently associated to $\mathrm{PH}, \mathrm{SICH}$, or SAH. Mortality at 90 days was lower in group 1 at $9.9 \%$, compared to $29.6 \%$ in group 3 $(p<0.001)$ and $20.7 \%$ in group $2(p=0.052)$. After adjusting for all relevant variables in the multivariate analysis, the difference was no longer significant (Table 3b).

Recanalization in group 1 was assessed with DSA in $97.5 \%$ of patients and with contrast-MRA or CTA in $2.4 \%$ (both basilar occlusions, one with CTA, as mTICI2b scored, and one with CE-MRA, as TICI3 scored); in groups 2 and 3 was assessed with DSA in $100 \%$. Regarding the pattern of recanalization in the groups, there were no significant differences in the rate of successful recanalization, taken as mTICI grade $2 \mathrm{~b}$ or 3 . In group 1 , only $18.5 \%$ of patients $(n=$ 15) achieved a mTICI 3 - complete recanalization, which was significantly lower than group 2 and 3 's equivalent rates of, respectively, 60.7 and $57.1 \%$ mTICI 3 recanalization $(p<$ 0.001 ). Patients with ELVOs that recanalized only with IVT had lower chance of achieving a complete recanalization (mTICI 3) compared to patients with ELVOs receiving
mTE/aTE with previous IVT (group 2, OR 0.15 [95\% CI 0.08-0.29]) or without (group 3, OR 0.17 [95\% CI 0.09$0.32]$ ). Conversely, group 1 had a sixfold chance of achieving an incomplete recanalization (mTICI $0-2 b$ ), compared to group 2, OR 6.67 (95\% CI 3.45-12.50), and compared to group 3, OR 5.88 (95\% CI 3.13-11.11), $p<0.001$ (Table 3c).

Receiving IV rtPA with or without mTE/aTE (patients from groups 1 and 2) led to mTICI3 in $43.8 \%$ compared to patients in group 3, which did not receive IVT, $57.1 \%$ (OR 0.59, 95\% CI 0.40-0.86; $p=0.007$ ).

The reasons for leaving a non mTICI 3 recanalization score in 66 patients of group 1 are elucidated in the following:

In 42 patients (63.6\%), the residual occlusion was not easily accessible for endovascular treatment producing a cuneiform perfusion defect distal to the occluded vessel. This defect was scored in 37 patients as recanalization pattern mTICI $2 b$. In 2 patients with a M2-3 occlusion in CTA, this remained exactly or almost exactly the same after IVT and scored mTICI 0 in 1 patient and mTICI 1 in the other patient. Following IVT, an MCA occlusion in the M1-segment in 2 patients turned into an occlusion in the truncus superior in 1 patient and the truncus inferior in the other patient with collaterals, scoring mTICI2a. One $\mathrm{T}$ occlusion ended up with distal embolisms in the anterior cerebral artery (A2-segment) and in the distal M2-segment (parietal posterior, noneloquent) with peripheral emboli in the entire MCA territory; this was scored as mTICI $2 \mathrm{a}$. 
Table 3. Multivariate analysis

\begin{tabular}{|c|c|c|c|}
\hline & OR & $95 \% \mathrm{CI}$ & $p$ value* \\
\hline \multicolumn{4}{|c|}{ a Clinical outcome $m R S 90$ days $0-2$ vs. $3-6(\mathrm{n}=363)$} \\
\hline Group 1: IVT vs. group 2: IVT + mTE/aTE & 1.16 & $0.50-2.71$ & 0.728 \\
\hline Group 1: IVT vs. group 3: mTE/aTE & 1.34 & $0.64-2.81$ & 0.439 \\
\hline Female vs. male & 0.58 & $0.34-0.98$ & 0.042 \\
\hline $\mathrm{DM}$ & 0.53 & $0.29-0.99$ & 0.046 \\
\hline SAH & 0.27 & $0.10-0.71$ & 0.008 \\
\hline Age, years & 0.94 & $0.92-0.97$ & $<0.001$ \\
\hline NIHSS & 0.88 & $0.85-0.92$ & $<0.001$ \\
\hline ASPECTS & 1.32 & $1.11-1.58$ & 0.002 \\
\hline \multicolumn{4}{|l|}{ b Mortality at 90 days $(\mathrm{n}=363)$} \\
\hline Group 1: IVT vs. group 2: IVT + mTE/aTE & 0.95 & $0.29-3.13$ & 0.931 \\
\hline Group 1: IVT vs. group 3: mTE/aTE & 0.47 & $0.15-1.46$ & 0.189 \\
\hline Age, years & 1.07 & $1.03-1.10$ & $<0.001$ \\
\hline NIHSS & 1.12 & $1.07-1.17$ & $<0.001$ \\
\hline ASPECTS & 0.75 & $0.65-0.86$ & $<0.001$ \\
\hline \multicolumn{4}{|l|}{ c Complete reperfusion $(\mathrm{n}=429)$} \\
\hline \multicolumn{4}{|l|}{ Group 1: IVT vs. group 2: IVT + mTE/aTE } \\
\hline mTICI 3 vs. mTICI $0-2 b$ & 0.15 & $0.08-0.29$ & $<0.001$ \\
\hline mTICI $0-2 b$ vs. 3 & 6.67 & $3.45-12.50$ & $<0.001$ \\
\hline \multicolumn{4}{|l|}{ Group 1: IVT vs. group 3: mTE/aTE } \\
\hline mTICI 3 vs. mTICI $0-2 b$ & 0.17 & $0.09-0.32$ & $<0.001$ \\
\hline mTICI $0-2 b$ vs. 3 & 5.88 & $3.13-11.11$ & $<0.001$ \\
\hline \multicolumn{4}{|c|}{$\begin{array}{l}\text { * Statistics: logistic regression with forward selection. } \\
\text { DM, diabetes mellitus; SAH, subarachnoid hemorrhage; ASPECTS, Alberta Stroke Program Early Computed } \\
\text { Tomography Score; IVT, intravenous thrombolysis; IVT + mTE/aTE, intravenous thrombolysis and subsequent- } \\
\text { ly mechanical and/or aspiration thrombectomy; NIHSS, National Institute of Health Stroke Scale; mRS, modified } \\
\text { Rankin Scale; mTE/aTE, mechanical and/or aspiration thrombectomy; mTICI, modified treatment in cerebral } \\
\text { ischemia score. }\end{array}$} \\
\hline
\end{tabular}

In 19 patients (28\%), there were peripheral emboli with no cuneiform defect, corresponding to mTICI $2 \mathrm{~b}$.

In 3 patients, there was a tandem occlusion, and after IVT persisting extracranial occlusion, and intracranial occlusion, which recanalized after IVT or with good leptomeningeal collaterals achieving a grade mTICI $2 \mathrm{~b}$.

Two patients with a basilar occlusion after IVT eventually achieved mTICI $2 \mathrm{~b}$ containing a small non-occlusive thrombus.

\section{Discussion/Conclusion}

According to the current guidelines, rtPA should be offered to all eligible patients with ELVO before attempting endovascular treatment. In patients under consideration for mTE, observation after IV alteplase to assess for clinical response should not be performed.
Patients should be transferred as fast as possible, in spite of concerns about a futile transfer [5]. In our study, clinical status after IVT is not a reliable predictor of successful recanalization in patients who had experienced recanalization after just IVT; a clinical improvement was seen in only $29.6 \%$ of these patients. In the literature, $41 \%$ of the interhospital transfers were reported to have been futile [6], with $48 \%$ of these patients showing either clinical improvement or arterial recanalization; this equals $20 \%$ of total transfers, highlighting that IVT administration cannot predict either clinical improvement or arterial recanalization after interhospital transfer [6].

In our study, patients that recanalized only with IVT had a lower NIHSS prior to angiography in comparison to the groups that received adjunctive thrombectomy, this is probably because the recanalization had already occurred. 
In our population, IVT with alteplase caused a successful or sufficient reperfusion in $10.6 \%$ of patients with ELVO referred to endovascular treatment. This low rate emphasizes the need of endovascular treatment in patients with ELVO receiving IVT. According to a previous meta-analysis of randomized controlled trials and observational cohorts, successful recanalization (mTICI $2 \mathrm{~b} / 3$ or significant clinical improvement suggestive of successful recanalization) after IVT prior to endovascular treatment occurs in 11\% (95\% CI 7-16\%) of patients with ELVOs and in studies which exclude tandem occlusions, this occurs in $17 \%$ of patients, $(95 \%$ CI 11-23\%) [1]. Series only focusing in patients treated with IVT report a much higher recanalization rate of $20 \%$ (95\% CI 15-26), varying according to occlusion site: this has been reported to be $38 \%$ in the distal MCA region, 35\% in the proximal MCA, 13\% in the ICA, and $13 \%$ in the basilar artery [7]. The variability in the rates could be explained by the time of measurement of recanalization, the use of different recanalization scales (e.g., report of recanalization of occluded vessel and not reperfusion rate or use of original TICI scale vs. mTICI scale), and diagnostic tools to assess reperfusion (e.g., transcranial Doppler or TOF-angiography might underdiagnose distal embolisms). The lower recanalization rate of IVT in our stroke population and also in the aforementioned meta-analysis [1] is probably due to the underreporting of patients that have an early improvement after IVT and therefore remain in their original hospitals. Another cause could be the fact that direct presenters with ELVO in centers that offer thrombectomy also receive prior IVT, in which rtPA has less time to work, or they do not receive the complete doses. Recanalization after IVT occurred in patients who were direct presenters in our center only in $1.3 \%$, much lower than recanalization with only IVT in patients transferred from other centers, 9.3\%, in which rtPA had more time to work.

Patients with ELVO that recanalize after just IVT tend to achieve a very high functional independence rate at 90 days; this was the case in $61.7 \%$ of the patients. Recent meta-analysis of 5 randomized controlled studies (stent-based thrombectomy versus IVT) [8], considering functional outcome in patients with ELVO treated only with IVT showed functional independence at 90 days in $26.46 \%$ of the patients. This also includes the patients that did not experience recanalization, which explains the lower functional independence rate compared to ours. In our own dataset, the mortality and SICH rates of patients with ELVOs, which recana- lized after just IVT were also lower (9.9 and 3.3\%, respectively), than in patients treated with just IVT in the meta-analysis (18.7 and 8.6\%, respectively) [8]. Although a direct comparison is not possible, the functional outcome of our group 1 was better, and mortality and SICH lower than in the thrombectomy group in the meta-analysis [8], in which $46.1 \%$ experienced independence, $15.3 \%$ mortality, and $7.8 \%$ SICH. In comparison, our patients with ELVO that recanalized after just IVT had a lower average stroke severity score (NIHSS 9 vs. 16.4 in the reported meta-analysis), most had an MCA occlusion that generally has a better recanalization rate [7] and also had higher ASPECTS and shorter time to IVT, both of which associated with an expected good clinical outcome. In our study, groups 2 and 3 represent older patients who generally had a worse mRS pre stroke, lower ASPECTS, more tandem occlusions, or carotid T occlusions, which are known to recanalize later. All those factors influence the clinical outcomes and this all goes toward explaining why their rate of independence at 90 days is lower than group 1 . After adjusting for all these variables, ELVO recanalization through IVT alone was not independently associated with a higher chance of a better outcome compared to endovascular treatment with or without previous IVT in our population.

In our study, patients with ELVO that recanalize with only IVT achieve a very high rate of successful recanalization and reperfusion, mTICI2b/3 (in 92.6\%), most of them mTICI2b. With regard to successful recanalization and reperfusion rate $(\mathrm{mTICI} 2 \mathrm{~b} / 3)$, there were no differences between groups 2 and 3 and group 1 in our study. However, achieving a complete reperfusion of mTICI 3 is less likely if an ELVO recanalizes after just IVT. The likelihood of a complete reperfusion of mTICI 3 is higher if recanalization was caused by $\mathrm{mTE} / \mathrm{aTE}$ with or without bridging with IVT.

The purpose of a thrombectomy maneuver is to remove the complete intracranial thrombus under proximal aspiration, reducing the risk of distal emboli in the previously ischemic territory of the occluded target artery. On the contrary, IVT causes lysis and fragmentation of the thrombus, dislodging smaller thrombus into smaller distal vessels where the lysis continues. In patients with ELVO that recanalizes only through IVT, our study observes that although IVT can achieve a proximal recanalization, a distal recanalization produces a cuneiform defect in $63.6 \%$ of cases or peripheral embolisms without cuneiform defect in $28 \%$. However, these perfusion defects do not seem to be associated with a 
worse clinical outcome in our study, as measured with mRS at 90 days.

Nowadays, some controversies exist as to whether IVT prior to mTE, "bridging," versus mTE alone are similar [9] or bridging IVT before mTE is superior [10]. Bridging may be associated with facilitating a thrombectomy and with lysis of distal thrombi [11]. These effects are occlusion site-dependent, easier thrombectomy (favored successful recanalization and first pass success) in distal, but not in proximal, MCA occlusions, with lower rate of complete reperfusion (TICI 3) [12]. In our study, IVT prior or not to $\mathrm{mTE} / \mathrm{aTE}$ was not independently associated to clinical outcome, safety variables, or successful recanalization rate; however, mTICI 3 was less frequently achieved.

The technical goal of a thrombectomy procedure should be a TICI grade $2 \mathrm{~b} / 3$ angiographic result [5]; however, successful reperfusion of mTICI 3 is associated with greater neurological improvement during hospitalization and better functional outcome at 90 days in comparison to mTICI $2 \mathrm{~b}$ and reduced infarct growth [13-15]. According to this, although a good functional outcome measured by mRS at 90 days was observed in our study in patients with ELVO that recanalized after just IVT, this resulted less frequent in a mTICI 3 reperfusion, the real prognosis is unknown and other deficits - not measured by mRS - could be possible, such as cognitive impairment, as has been reported from microembolization following carotid interventions [16].

Our study does not pretend to be a case - control study, but aimed to describe characteristics of patients with ELVO that recanalize with only IVT and to compare them with a group of patients with ELVO treated in "real clinical practice," outside limitations of clinical trials (mRS pre stroke, lower basal ASPECTS, longer time to intervention, and so on); multivariate analysis was done to adjust all those imbalances. To our knowledge, this is the first report of detailed analysis of reperfusion in patients with an ELVO recanalizing after just IVT and suggests that assuming a complete reperfusion of a proximal ELVO in these patients by only considering clinical improvement or the recanalization of the proximal ELVO is not a real measure of reperfusion. Incomplete reperfusion is more frequent in patients with an ELVO with recanalization after just IVT. A score mTICI2b, also considered a successful recanalization, has no translation in $\mathrm{mRS}$ at 90 days, but the meaning of distal peripheral emboli or a cuneiform perfusion defect should not be underestimated or neglected. The limitations of our study include an un- derestimation of recanalization rate by IVT caused by underreporting of patients with very early neurological improvement. An incomplete dose of IVT given in 5\% of patients in group 1, and $19.7 \%$ of patients of group 2 could also underestimate the reperfusion rate.

In conclusion, pretreating an ELVO with IVT may cause a recanalization in $10.6 \%$ of cases, making endovascular treatment unnecessary. Early recanalization of ELVO with only IVT is associated with a very high functional independence rate at 90 days and similar successful reperfusion rates (mTICI2b/3) compared to ELVO treated with endovascular treatment, with or without previous IVT. However, recanalization only through IVT achieves a lower rate of mTICI 3 reperfusion compared to endovascular treatment.

\section{Acknowledgments}

The authors are most grateful to M. Kuhnert for language revision of the manuscript; H. Niggemann for her support in statistical analysis, and C. Knispel, stroke and study nurse of the Neuroradiological Clinic for participating in data collection and data maintenance.

\section{Statement of Ethics}

The local Ethics Committee approved the retrospective data collection and analysis.

\section{Disclosure Statement}

M.A.P. and V.H. have a consultation and proctoring agreement with phenox GmbH. H.H. is cofounder and a shareholder of phenox $\mathrm{GmbH}$; the other authors report no conflict of interest.

\section{Funding Sources}

None.

\section{Author Contributions}

C.S.: study concept, acquisition, analysis, interpreting of data, and drafting of the manuscript. M.A.P., V.H., and M.A.: contributed to the acquisition of data. H.B. and H.H.: study concept, interpreting of data, drafting of the manuscript, critical revision, and final approval of the manuscript. 


\section{References}

1 Tsivgoulis G, Katsanos AH, Schellinger PD, Köhrmann M, Varelas P, Magoufis G, et al. Successful Reperfusion With Intravenous Thrombolysis Preceding Mechanical Thrombectomy in Large-Vessel Occlusions. Stroke. 2018 Jan;49(1):232-5.

2 Zaidat OO, Yoo AJ, Khatri P, Tomsick TA, von Kummer R, Saver JL, et al.; Cerebral Angiographic Revascularization Grading (CARG) Collaborators; STIR Revascularization working group; STIR Thrombolysis in Cerebral Infarction (TICI) Task Force. Recommendations on angiographic revascularization grading standards for acute ischemic stroke: a consensus statement. Stroke. 2013 Sep;44(9):2650-63.

3 Saver JL. Novel end point analytic techniques and interpreting shifts across the entire range of outcome scales in acute stroke trials. Stroke. 2007 Nov;38(11):3055-62.

4 Hacke W, Kaste M, Fieschi C, von Kummer R, Davalos A, Meier D, et al.; Second European-Australasian Acute Stroke Study Investigators. Randomised double-blind placebocontrolled trial of thrombolytic therapy with intravenous alteplase in acute ischaemic stroke (ECASS II). Lancet. 1998 Oct; 352(9136):1245-51.

5 Powers WJ, Rabinstein AA, Ackerson T, Adeoye OM, Bambakidis NC, Becker K, et al.; American Heart Association Stroke Council. 2018 Guidelines for the Early Management of Patients With Acute Ischemic Stroke: A Guideline for Healthcare Professionals From the American Heart Association/American Stroke Association. Stroke. 2018 Mar;49(3): e46-110.

6 Fuentes B, Alonso de Leciñana M, XiménezCarrillo A, Martínez-Sánchez P, Cruz-Culebras A, Zapata-Wainberg G, et al.; Madrid Stroke Network. Futile Interhospital Transfer for Endovascular Treatment in Acute Ischemic Stroke: The Madrid Stroke Network Experience. Stroke. 2015 Aug;46(8):2156-61.

7 Seners P, Turc G, Maïer B, Mas JL, Oppenheim C, Baron JC. Incidence and Predictors of Early Recanalization After Intravenous Thrombolysis: A Systematic Review and Meta-Analysis. Stroke. 2016 Sep;47(9):2409-12.

8 Grech R, Schembri M, Thornton J. Stentbased thrombectomy versus intravenous tissue plasminogen activator in acute ischaemic stroke: A systematic review and meta-analysis. Interv Neuroradiol. 2015 Dec;21(6):68490.

9 Coutinho JM, Liebeskind DS, Slater LA, Nogueira RG, Clark W, Dávalos A, et al. Combined Intravenous Thrombolysis and Thrombectomy vs Thrombectomy Alone for Acute Ischemic Stroke: A Pooled Analysis of the SWIFT and STAR Studies. JAMA Neurol. 2017 Mar;74(3):268-74.

10 Mistry EA, Mistry AM, Nakawah MO, Chitale RV, James RF, Volpi JJ, et al. Mechanical Thrombectomy Outcomes With and Without Intravenous Thrombolysis in Stroke Patients: A Meta-Analysis. Stroke. 2017 Sep;48(9): $2450-6$.
11 Tsivgoulis G, Katsanos AH, Mavridis D, Alexandrov AW, Magoufis G, Arthur A, et al. Endovascular thrombectomy with or without systemic thrombolysis? Ther Adv Neurol Disorder. 2017 Mar;10(3):151-60.

12 Kaesmacher J, Kleine JF. Bridging Therapy with i.v. rtPA in MCA Occlusion Prior to Endovascular Thrombectomy: a Double-Edged Sword? Clin Neuroradiol. 2018 Mar;28(1): 81-9.

13 Goyal N, Tsivgoulis G, Frei D, Turk A, Baxter B, Froehler MT, et al. Comparative Safety and Efficacy of Modified TICI 2b and TICI 3 Reperfusion in Acute Ischemic Strokes Treated With Mechanical Thrombectomy. Neurosurgery. 2018 Sep;83(3):593.

14 Chamorro Á, Blasco J, López A, Amaro S, Román LS, Llull L, et al. Complete reperfusion is required for maximal benefits of mechanical thrombectomy in stroke patients. Sci Rep. 2017 Sep;7(1):11636.

15 Dargazanli C, Consoli A, Barral M, Labreuche J, Redjem H, Ciccio G, et al. Impact of Modified TICI 3 versus Modified TICI 2b Reperfusion Score to Predict Good Outcome following Endovascular Therapy. AJNR Am J Neuroradiol. 2017 Jan;38(1): 90-6.

16 Zhou W, Baughman BD, Soman S, Wintermark M, Lazzeroni LC, Hitchner E, et al. Volume of subclinical embolic infarct correlates to long-term cognitive changes after carotid revascularization. J Vasc Surg. 2017 Mar; 65(3):686-94. 